\title{
Paired structures in knowledge representation
}

\author{
J. Montero ${ }^{\mathrm{a}}$, H. Bustince ${ }^{\mathrm{b}}$, \\ C. Franco ${ }^{\mathrm{c}}$, J.T. Rodríguez ${ }^{\mathrm{d}}$, D. Gómez ${ }^{\mathrm{e}}$, \\ M. Pagola ${ }^{\mathrm{b}}$, J. Fernandez ${ }^{\mathrm{b}}$, E. Barrenechea ${ }^{\mathrm{b}}$ \\ ${ }^{\text {a }}$ Faculty of Mathematics, Complutense University, and Geosciences Institute IGEO (CSIC- \\ UCM), Plaza de Ciencias 3, Madrid 28040, Spain \\ monty@mat.ucm.es

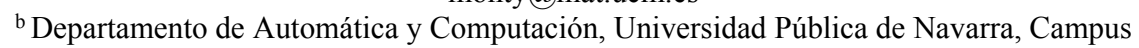 \\ Arrosadia s/n, 31006, Pamplona, Spain \\ \{bustince, edurne.barrenechea, fcojavier.fernandez, miguel.pagola\}@unavarra.es \\ ${ }^{\mathrm{c}}$ IFRO, Faculty of Science, University of Copenhagen, Bülowsvej 17, 1870 Frederiksberg C, \\ Denmark \\ cf@ifro.ku.dk \\ ${ }^{\mathrm{d}}$ Faculty of Mathematics, Complutense University, Plaza de Ciencias 3, Madrid 28040, Spain \\ jtrodrig@mat.ucm.es \\ ${ }^{e}$ Faculty of Statistics, Complutense University, Av. Puerta de Hierro s/n, Madrid 28040, Spain \\ dagomez@estad.ucm.es
}

\begin{abstract}
In this position paper we propose a consistent and unifying view to all those basic knowledge representation models that are based on the existence of two somehow opposite fuzzy concepts. A number of these basic models can be found in fuzzy logic and multi-valued logic literature. Here it is claimed that it is the semantic relationship between two paired concepts what determines the emergence of different types of neutrality, namely indeterminacy, ambivalence and conflict, widely used under different frameworks (possibly under different names). It will be shown the potential relevance of paired structures, generated from two paired concepts together with their associated neutrality, all of them to be modelled as fuzzy sets. In this way, paired structures can be viewed as a standard basic model from which different models arise. This unifying view should therefore allow a deeper analysis of the relationships between several existing knowledge representation formalisms, providing a basis from which more expressive models can be later developed.
\end{abstract}

Keywords: Knowledge representation; Paired structures; Neutral concepts; Bipolarity.

\section{Introduction}

Recent advances in Psychology and Neurology are providing relevant results for the development of decision making models. The human brain has specifically and successfully evolved to manage complex, uncertain, incomplete, and even apparently inconsistent information. For example, neurologists have shown that the part of the brain taking care of making up the last decision is different to the part of the brain in charge of the previous rational analysis of alternatives, being the first part associated to emotions (see, e.g., [6],[7]). A number of similar results within neurology (see, e.g. 
[41],[52],[82]) suggest that the activation of different areas of the brain, associated with both cognition and emotion, participate in our decision processes through the continuous interplay among different networks (namely the valuation network, the control network and the memory system), each one following their own set of rules (see, e.g., [59],[60]). Among other key achievements, it has been recently shown the key role that concept representation plays in our knowledge process (see, e.g., [10],[39]), along with the fact that the human brain manages positive information in a different way than negative information. This observation suggests some kind of bipolarity in the way that our brain handles information (see, e.g., [17], [18]). Positive and negative affects are not processed in the same region of the brain, as they are generated by clearly different neural processes [61].

The importance of bipolar reasoning in human activity was emphasized by Osgood, Suci and Tannenbaum in 1957 [54] (see also [38], [71]). These authors proposed a semantic theory based on the Semantic Differential (SD) scale for evaluating the meaning of concepts. This theory became very popular for measuring attitudes in a practical way, where individuals are asked to use the SD scale to evaluate if a given object is perceived as being positive, neutral or negative.

Nonetheless, it becomes evident that by using the SD scale, objects cannot be evaluated as being positive and negative at the same time, and its neutral value can hardly be understood as a proper representation of neutrality. From this perspective, there are certain attitudes that seem to escape the linear logic of such a scale, but still require proper representation. This led to some critiques (see, e.g., [18], [27], [38]), stating that the SD scale does not consider other relevant attitudes arising from the inherent tension among opposite-like concepts, like for example ambivalence. Hence, a modified SD scale was proposed (see, e.g., [38]), consisting of two unipolar scales joined together by their minimal element, allowing the simultaneous measurement of positive and negative evaluations.

The relevance of this discussion can be well positioned and illustrated by different works in multicriteria decision making and decision theory (see e.g. [36], [37], [81]). There, the SD scale, or unipolar bivariate model [22], [36], has been widely applied, and further developed into more complex scales. These scales are grouped together under a general (somewhat oversimplifying) category of bipolar univariate models (for some examples on opposite-based decision modeling see again [36], but also [31], [78]).

Moreover, it can be stated that our internal decision making process is of a complex nature, implying previous differentiated knowledge acquisition and representation processes (see, e.g., [48], [49]), quite often based upon multi-criteria arguments. In fact, the linear logic behind the SD scale does not allow representing the natural complexity we perceive from reality. Hence, once such a complexity is acknowledged, our mathematical modelling must continuously balance precision and simplicity, just as our brain looks for relevant but at the same time manageable information.

But whenever an objective measure for a concept is not available, it will be difficult to manipulate such a concept in an isolated manner. Most surely, immediately related concepts need to be taken into account. Generally speaking, understanding concepts by means of two opposite concepts, implies that we can capture the tension between both opposites. In some way, such simultaneous opposite views are unavoidable to start 
understanding the world, and indeed we need more complex knowledge representation structures to manage more than two views.

From our standpoint, most concepts cannot be properly understood in an isolated way. Addressing two different views seems to be the basic model to start with (although some concepts might need more than one surrounding concept in order to understand its limits). A number of quite similar fuzzy models focussing on the existence of two opposite concepts can be found in the literature, somehow offering a confusing view that we pretend to unify and explain within the unique umbrella of paired fuzzy sets and paired structures. We cannot understand certain concepts without understanding their opposite concepts. Pairs of predicates that will constitute paired concepts are, for example, tall/short, fat/slim, big/small, cheap/expensive or good/bad (see e.g. [63]).

The point of departure of this paper can be found in the above considerations, together with the bipolar approach proposed by Dubois and Prade in several papers (see [22], [23], [24]). Among other things, Dubois and Prade proposed a classification of bipolar models in three types of bipolarity that indeed shows similarities with our proposal below, but also essential differences: our approach, as it will be seen, follows from a constructive view of what we call paired structures, by focussing on how the semantic tension between two opposites generates certain types of neutrality (see [62] for a previous attempt). In this sense we emphasize the key role of certain neutralities in our knowledge representation models, as pointed out by Atanassov [4], Smarandache [70] and others. But notice that our notion of neutrality should not be confused with the neutral value in a traditional sense (see [22], [23], [24], [36], [54], among others). Instead, we will stress the existence of different kinds of neutrality that emerge (in the sense of [11]) from the semantic relation between two opposite concepts (and notice also that we refer to a neutral category that does not entail linearity between opposites). Such a constructive view establishes an alternative to Dubois-Prade's approach, providing a distinction of those models based on opposites different from their types of bipolarity. Moreover, the term paired concepts we propose instead is not subject to be confused with the term bipolarity in the sense of a psychological disorder.

Therefore, our alternative for modelling basic knowledge representation is based on paired concepts, which will naturally lead to paired structures. A paired structure is defined by a pair of opposite concepts plus their associated neutralities and the relationships between these elements. Such a basic structure stands as a primary foundation from where further valuation scales and learning processes can be developed. As a consequence, it can be understood as a first stage for more complex and meaningful evaluation structures, where non-neutralities are allowed besides the original two opposites. This paired approach has already led to a specific model for preference representation (see [32]), a particular case whose general framework should be found in this paper.

Let us remark that this paper is not about formal logic or its interpretation. It rather deals with knowledge and natural language representation by means of logical tools.

In order to illustrate our position, this paper is organized as follows: in the next section we shall present a general example from where our discussion will evolve. Our proposal will be formalized in Section 3, restricted to our definition of opposite concepts. From this definition we shall formalize what we understand by paired fuzzy sets and paired structures. We shall expose the types of neutrality that rise from paired fuzzy sets, and that will produce different paired structures. Section 4 is devoted to 
compare our proposal with some related existing models. A discussion in Section 5 shows a standard procedure for building paired structures, and a final Section 6 is devoted to discuss some open key issues for future research.

\section{Preliminary Example: On the Representation and Measurement of Size}

Let us try to illustrate our view through a classical well-known example.

The meaning of the notion size of a person can be modelled in terms of predicates defining an evaluation scale. The structure of such a scale highly depends on how size is perceived, and particularly on whether it is viewed as a 1-dimensional or multidimensional characteristic. For example, in case size is understood as size $=$ height, the verification of its occurrence can be evaluated within a linear scale. Let us examine more in detail this meaning of size = height .

Although we all know that height is measurable in the real line, we should realize that we usually do not try to measure the height of each person we meet with a value in the real line. Instead of saying "Paula's height looks around 1'90 meters", most people will talk about Paula as a tall person, i.e., in terms of the tallness concept, which can be regarded as a fuzzy context-dependent concept [85]. Indeed, a person's height is usually judged in terms of the predicates tall and short, which constitute semantic references or landmarks for the evaluation of such a notion. We hardly use the notion of a person's height without the landmarks provided by the opposites tall and short, or any other equivalent pair of opposite predicates.

If our concept of tallness were crisp, the sentence "Paula is tall" would have a direct translation on the evaluation scale in terms of height: for example, "Paula is tall" if and only if "Paula's height is at least 1'70 meters". As soon as we have this crisp definition, the concept of being non-tall is automatically created by the classical crisp negation: "Paula is non-tall" if and only if "Paula's height is less than 1'70 meters". That is,

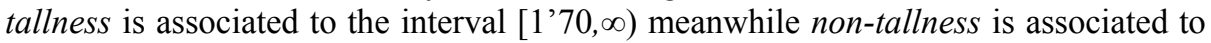
the interval $\left(0,1^{\prime} 70\right)$. In order to generate such paired predicates (tall and non-tall), we simply need to assume the existence of the crisp negation: a person $x$ within a community $X$ belongs to the set of tall people if and only if the height $h(x)$ of such a person is greater than or equal to 1'70. And a person $x$ within the community $X$ belongs to the set of non-tall people within $X$ if and only if the height $h(x)$ of such a person is smaller than 1'70. The set of tall people is Tall $=\left\{x \in X, h(x) \geq 1^{\prime} 70\right\}$, and the set of non-tall people is Non-tall $=\left\{x \in X, h(x)<1^{\prime} 70\right\}$. Within such a crisp context, no person can be tall and non-tall at the same time, and everybody will be either tall or non-tall. A basic paired structure to represent height has been built from only one predicate (tall) and its negation (non-tall), and the characteristic functions of both crisp predicates

$$
\mu_{\text {Tall }}(x)=1 \Leftrightarrow h(x) \geq 1^{\prime} 70, \quad \mu_{\text {Tall }}(x)=0 \Leftrightarrow h(x)<1^{\prime} 70
$$

are defined in such a way that

$$
\begin{aligned}
& \mu_{\text {Tall }}(x)=n\left(\mu_{\text {Non-tall }}(x)\right), \forall x \in X \\
& \mu_{\text {Non-tall }}(x)=n\left(\mu_{\text {Tall }}(x)\right), \forall x \in X
\end{aligned}
$$


being $n:\{0,1\} \rightarrow\{0,1\}$, such that $n(0)=1$ and $n(1)=0$, the only negation within the crisp $\{0,1\}$ framework (in fact, and within the crisp framework, the only one-to-one mapping from $\{0,1\}$ into itself different from the identity mapping). These are well known facts, but it is important to show that tall and non-tall appear as paired predicates in a natural way within the crisp framework. Meanwhile a measurement of height is available and precise, there is no room for any kind of neutral concept. Borders between a predicate and its negation ( $\{x \in X, h(x)=1$ ' 70$\}$ in the above example) might still deserve specific attention, precisely because of a potential estimation uncertainty.

However, the introduction of short as the opposite predicate of tall allows different translations into a measurable height, being tall and short a different couple of paired predicates than tall and non-tall.

For example, and keeping the crisp approach, we can define that "Paula is short" if and only if "Paula's height is at most 1'60 meters". Again, the concept of non-short is automatically created, in such a way that Short $=\left\{x \mid x \in X, h(x) \leq 1^{\prime} 60\right\}$, and the set of non-short people is Non-short $=\left\{x \mid x \in X, h(x)>1^{\prime} 60\right\}$. In this way, all those people with height within the interval (1'60,1'70) are neither tall nor short, leading to some kind of indeterminacy (we cannot assign any of the only two available predicates to some individuals). In this particular case we know that, in order to solve this indeterminacy, we can create an intermediate non-neutral concept, like Medium $=\{x \mid$ $\left.x \in X, 1^{\prime} 60<h(x)<1^{\prime} 70\right\}$. But this is a different kind of argument, to be conducted in a subsequent stage. At first, what we find is just that none of the two opposite, available predicates apply to some individuals. In general, such indeterminacy suggests the need for a new predicate. Such a new predicate will not necessarily be a non-neutral intermediate predicate as Medium in this example, where a non-paired three-valued linear scale Tall-Medium-Short is easily suggested (please be aware of the specificity of this example, where the linear representation behind is a priori known and the two opposite predicates we have chosen correspond to left and right tails).

On the other hand, we could have defined that "Paula is short" if and only if "Paula's height is at most 1'80 meters". In this case, Short $=\{x \mid x \in X, h(x) \leq 1$ ' 80$\}$. Then all those people with height within the interval [1'70,1'80] will be both tall and short, leading to a certain ambivalence. That is, what we find now is that both opposite predicates simultaneously apply. Clearly, in this specific example it is again suggested an intermediate concept in between opposites, like Medium $=\left\{x \mid x \in X, 1{ }^{\prime} 70 \leq h(x) \leq\right.$ $\left.1^{\prime} 80\right\}$, and then we could reshape opposites to avoid overlapping (for example by redefining Tall $=\{x \mid x \in X, h(x)>1$ ' 80$\}$ and Short $=\{x \mid x \in X, h(x)<1$ '70 $\})$. Once more, the construction of a linear evaluation scale (or equivalently, the interpretation of such ambivalence as an intermediate predicate Medium) depends on a subsequent different kind of argument relying on a previous interpretation of the opposites and their relationship. But at a first stage we can only acknowledge whether there are persons being both tall and short (ambivalence) or none of those predicates apply to some people (indeterminacy).

Here it becomes evident the following observation, quite standard within a classification framework (see, e.g., [1], [2]): two opposite crisp predicates (tall/short) that refer to the same notion (height) can generate different neutral concepts (indeterminacy and ambivalence, or both), depending on their semantics. A more careful analysis of indeterminacy and ambivalence might suggest specific scales (see, e.g., [40]) by modifying the definition of the two basic opposite predicates and/or 
introducing new intermediate predicates. But these evaluation scales and their corresponding semantics can only be properly understood by firstly addressing the particular semantic relationship between the two basic opposites.

A quite similar situation can be found within logic and the classical square of oppositions, where it is understood that a concept $p$ comes with its negation $n(p)$ and its antonym ant $(p)$, being $p$ and ant(p) mutually exclusive contraries (allowing therefore indeterminacy). In this way, $n(p)$ and $n($ ant $(p))$ are overlapping $s u b$-contraries (see [25]). But notice that, following Amo et al. [2], we should avoid a priori artificial conditions. In practice, such properties will be eventually reached after a sometimes long learning process: in our model, a concept should not be initially associated to a contrary, but simply to a more general opposite. Depending on the semantic relationship between the initial concept and its opposite, we shall be able to identify indeterminacy, overlapping or any other kind of neutrality. Moreover, notice that different neutralities can simultaneously appear (for example, the selected opposite of rather tall is naturally rather short, and indeterminacy arising from these two opposites should not be simply associated to a middle stage between extremes).

In particular, in addition to indeterminacy and ambivalence, there is a third standard neutrality that can appear in more complex situations. In the above example we have based our arguments upon the existence of a unique, linearly-based property for understanding size, given by height. In practice, however, most of our concepts are complex in the sense that they can be explained in terms of a number of simpler concepts (see, e.g., [32]). In this case, our evaluation proceeds through a (perhaps non conscious) aggregation process.

For example, when talking about the size of a person we can define two complex opposite predicates like big and small. But being big or small might depend on height (tall versus short) and weight (fat versus slim). Of course it may be the case that a person is neither big nor small, being there indeterminacy. And another person can be both big and small, being there ambivalence. But it can also happen that we cannot choose among big and small because there is a conflict behind, i.e. both opposite predicates hold in a conflictive manner. It is not the same to say that a person is both big and small because s/he is simultaneously both tall and short, or both fat and slim, than to say that such a person is big and small because s/he is tall but slim, or fat but short. The taller and fatter a person is, the bigger such a person is; the shorter and the slimmer a person is, the smaller such a person is. But taller and slimmer are conflictive arguments, like shorter and fatter are. In this more complex (multicriteria) framework we can find conflict as a third kind of neutrality associated to opposite complex predicates, in addition to indeterminacy and ambivalence. Similitude of these conflictive situations with incomparability within preference modelling is obvious since they share an underlying multicriteria view (see, e.g., [29], [30] and [78]). But conflict is the cognition we detect, not any specific decision making solution.

To conclude this example, let us briefly discuss the role of points of symmetry. To this end, let us assume again that the meaning of size is interpreted solely in terms of the measurable characteristic height, and that our references are given by the predicates tall and not-tall. Let us now consider these as fuzzy (i.e. non-crisp) concepts. That is, we now allow both predicates to be evaluated on the interval $[0,1]$ rather than on the binary set $\{0,1\}$. Then, for any $x \in X$, we may for instance set the meaning of tall and its complement to be represented by the fuzzy sets 


$$
\mu_{\text {Tall }}(x)= \begin{cases}0 & \text { if } h(x)<1^{\prime} 70 \\ \frac{h(x)-1^{\prime} 70}{1^{\prime} 80-1^{\prime} 70} & \text { if } 1^{\prime} 70 \leq h(x) \leq 1^{\prime} 80 \quad, \quad \mu_{\text {Not-tall }}(x)=1-\mu_{\text {Tall }}(x) . \\ 1 & \text { if } h(x)>1^{\prime} 80\end{cases}
$$

As usually admitted, this kind of representation enables both tall and its negation not-tall to exhibit an imprecise semantic, avoiding the boundary problems associated to crisp predicates. But, apart from that, we still have just a predicate and its negation as opposites, fully explaining the whole universe of discourse $X$. No room is left for neutral concepts. However, contrarily to the previous crisp context, now it is possible for an object to be equally associated to a concept and its opposite, as now $\mu_{\text {Tall }}(x)=\mu_{\text {Not-tall }}(x)=0.5$ can hold for a given object $x \in X$ (i.e., whenever $h(x)=1$ '75 in the current example). We refer to this situation by saying that, in a fuzzy context, a reference predicate and its negation admit a point of symmetry between them. It is then important to stress that such point of symmetry does not represent a new concept: as exposed above in the crisp case, there are no other available options besides the opposites, as both predicates are related by negation.

In this way we can see how the tension between our two initial opposite predicates suggests certain neutral categories and, eventually, a particular reshaping of those opposite predicates (or alternatively, a non-paired structure). Non overlapping opposites might produce indeterminacy, overlapping opposites produce ambivalence, and multi-dimensional opposites might produce conflict.

\section{Semantic opposites and paired structures}

Let us study the relationships that can arise between a pair of predicates when these two predicates are semantically opposite, constituting the reference landmarks of a linguistic representation of reality. We will refer to those pairs of opposite predicates as paired concepts. Two paired concepts can generate neutral concepts, and meanwhile we consider only paired concepts together with associated neutral concepts we shall have a paired structure.

\subsection{Paired concepts}

Paired concepts are not simply a couple of predicates. Our first objective is to clarify what we mean by paired. Paired predicates must be specifically related. To begin the discussion about the nature of this relationship, let us remind that, as pointed out above, our mind is able to represent complex situations, related to interests and emotions, by using a pair of landmarks that constitute the references for evaluation. Such reference concepts allow configuring the evaluation framework in which information can be assessed. In other words, they constitute the referential context in which pieces of information are understood. As shown in our preliminary example, only if tallness can be known by means of a specific measure we can be confused in thinking that tallness can be understood without an opposite predicate like shortness. Otherwise, if such a 
measure has not yet been provided, even the predicate tallness requires an opposite predicate like shortness in order to be understood.

We cannot understand most predicates without also knowing the meaning of all those other predicates that define their limits (a complex concept might need several additional predicates to show its limits). In this sense, two predicates have to be related in some specific way to effectively configure an appropriate referential context, i.e., in order to properly constitute a pair of reference landmarks. The previous arguments suggest the existence of a particular structure, in the sense of a set of objects (predicates) together with a set of relationships (opposition, neutrality, etc.) between them.

Our point is that we should focus on the semantic opposition between paired predicates (see, e.g., [78]). However, such an opposition does not have a unique possible representation or definition, and from the different opposition relationships different neutral valuation concepts will emerge. For example, very tall and very short are opposite predicates, and more or less tall and more or less short are also opposite predicates, but they indeed suggest very different spaces in between them In this way, we will refer to negation, antonym and sub-antonym to capture the basic possibilities for such a semantic opposition.

\subsection{Antonym and sub-antonym with respect to a negation}

In this paper, we focus on three particular forms of such a semantic opposition between predicates, specifically negation, antonym and sub-antonym (see e.g. [72], [75], but also [64]).

Before formalizing these three basic semantic relationships, it is important to make explicit that we assume that any predicate $P$ (and thus particularly our reference opposites) can be represented as a fuzzy set $\mu_{P}$ over a particular universe of discourse $X$. In this way, $\mu_{P}(x) \in[0,1]$ denotes the degree up to which an object $x \in X$ verifies predicate $P$. Hence, the membership function $\mu_{P}$ is taken to represent the semantics (i.e. the practical meaning) of the predicate $P$ in the context given by the universe $X$.

In a first approach, we will also assume reference predicates to be simple, in the sense of referring to a characteristic depending on just a single criterion or dimension (like tall and short refer to size = height), i.e. not admitting a further decomposition in a set of underlying criteria or sub-predicates. As already pointed out, we can later on remove this assumption and analyze also complex multidimensional reference concepts, as could be big/small or good/bad (these concepts usually require a further explanation in terms of a set of underlying criteria).

Now, let us recall that a negation function within the fuzzy context (see again [72], [75]) is usually understood as a non-increasing function $n:[0,1] \rightarrow[0,1]$ such that $n(0)$ $=1$ and $n(1)=0$. All throughout this paper we are assuming that such a negation is a strong negation, i.e., a strictly decreasing, continuous negation being also involutive (i.e. such that $n(n(v))=v$ for all $v$ in [0,1]). If $F(X)$ denotes the set of all fuzzy sets (i.e. predicates) over a given universe $X$, then any strong negation function $n$ determines a negation operator $N: F(X) \rightarrow F(X)$ such that $N(\mu)(x)=n(\mu(x))$ for any predicate 
$\mu \in F(X)$ and any object $x \in X$. It is therefore assured that our negation operator $N: F(X) \rightarrow F(X)$ verifies

A1) $N$ is involutive (i.e., the negation of the negation is the identity);

A2) $\mu(x) \leq \mu(y) \Rightarrow N(\mu)(y) \leq N(\mu)(x)$ for all $\mu \in F(X)$ and $x, y \in X$;

Any operator $O: F(X) \rightarrow F(X)$ verifying these two properties $\mathrm{A} 1$ (it is involutive in the sense that the opposition of the opposition is the identity) and A2 (it is monotonous) will be called an opposition operator.

Let us assume now that a particular negation operator $N: F(X) \rightarrow F(X)$ (or equivalently a particular negation function $n:[0,1] \rightarrow[0,1])$ has been fixed. An antonym operator was then defined in [76] as a mapping $A: F(X) \rightarrow F(X)$ verifying

A1) A is involutive (i.e., the antonym of the antonym is the identity);

A2) $\mu(x) \leq \mu(y) \Rightarrow A(\mu)(y) \leq A(\mu)(x)$ for all $\mu \in F(X)$ and $x, y \in X$;

A3) $A \leq N$.

In other words, an antonym is a particular opposition verifying condition $\mathrm{A} 3$, to be associated to a contrary within the nomenclature of the logical square of oppositions (see [25]).

It seems therefore natural to define a sub-antonym operator (to be associated to a sub-contrary within the nomenclature of square of oppositions [9], [25], [51], [53]) as a mapping $A: F(X) \rightarrow F(X)$ that fulfils

A1) A is involutive (i.e., the sub-antonym of the sub-antonym is the identity);

A2) $\mu(x) \leq \mu(y) \Rightarrow A(\mu)(y) \leq A(\mu)(x)$ for any $\mu \in F(X)$ and $x, y \in X$.

A4) $A \geq N$.

Hence, $N$ is in fact the only operator being simultaneously an antonym and a subantonym with respect to $N$. Both antonyms and sub-antonyms are instances of opposition operators, and there are oppositions being neither an antonym nor a subantonym. In the following we shall refer to antonym as any antonym not being the given negation $N$. Analogously, we shall refer to sub-antonym as any sub-antonym not being the given negation $N$.

In a general sense, we consider that two predicates (or fuzzy sets) $P, Q$ are paired if and only if $P=O(Q)$, and thus also $Q=O(P)$, holds for a certain semantic opposition operator $O$. Our point is that neutral predicates emerge from the specific relation of opposition holding between paired concepts.

\subsection{The concept of neutrality}


Neutral concepts are generated from two opposite predicates whenever an object cannot be properly explained in terms of both opposites. From a fuzzy approach, the point here is that the two opposites may not define a fuzzy partition of the universe of discourse, see [66] or any of its generalizations based upon any alternative logic (e.g. [19], but particularly [1], [2]). In this context, the term neutral represents an additional valuation concept that is being semantically generated from opposites. As suggested in [48], different types of neutrality or neutral concepts can emerge from the tension between opposites (see also [11]).

In the above preliminary example, we started from the opposition between a concept and its negation, and then we considered antonym and sub-antonym concepts. In this way, two types of neutrality have been already introduced: indeterminacy and ambivalence. The first type of neutrality suggests that both opposite concepts overlap [14].The second type of neutrality suggests that objects cannot be fully explained solely in terms of the given opposites [16].

But we were in fact assuming that there was a unique underlying simple, 1dimensional criterion or characteristic. In many contexts, however, opposites are rather complex predicates (as good/bad, beautiful/ugly or the previous big/small). These complex predicates show a multidimensional nature and suggest an explanation in terms of simpler reference predicates. This situation can be associated to a multi-criteria framework, in which the verification of a concept is obtained through the aggregation of several criteria. It is in this context where conflict can naturally appear as a third type of neutrality. Such conflict should be naturally expected within complex paired structures, whenever serious arguments for both opposites are simultaneously found in different, independent criteria (like when in the big/small example we find that someone is simultaneously very tall, suggesting a big person, but very slim, suggesting a small person). This situation suggests that complex opposites can show a kind of conflictive relation, different to the ambivalence or the overlapping associated to opposite concepts over a simple, 1-dimensional characteristic. In fact, this conflict should not be expected when dealing with paired structures on a 1-dimensional argument. Of course, different kinds of conflict can be acknowledged in higher multidimensional problems besides the above conflict between two underlying criteria.

In summary, we should stress that different types of neutrality may appear depending on the semantic relationship between opposites. That is, it is the opposition relationship what determines the particular meaning of the different types of neutrality that may arise, and at the same time, of the whole paired structure. All these kinds of neutrality are quite often confused (sometimes even labelled under the same word) whenever a decision maker finds a kind of symmetrical situation that makes essentially difficult to choose among both opposites.

Such hesitation is suggested for the above neutralities, but there are also non-neutral situations that show another kind of hesitation. For example, imprecision when estimating degrees of membership implies facing a different kind of uncertainty, related to the difficulty of choosing an exact value. Hence, even if two paired concepts cover reality without indeterminacy, as happens when opposition is represented through a given negation, uncertainty associated to estimation uncertainty may still represent a specific kind of hesitation. However, such hesitation due to imprecision should not be considered as a neutral concept, as it is not generated from the semantic tension between opposites. 
An easy approach for this imprecision problem is to associate an interval instead of a single value (see, e.g., [16]). The wider such an interval is, the more imprecise our estimation. Maximum imprecision for a membership value will be then associated to the complete unit interval, which would mean that we simply do not have any useful information about such an estimation (see [68] for a complete approach within a probabilistic framework, and [67] for the seminal approach within a fuzzy context). In general, type-2 fuzzy sets [42] can naturally appear associated to this estimation problem.

\subsection{Paired structures}

Paired structures belong to a first level of knowledge representation. They stand as the subjacent semantic structure created from two opposite predicates and their associated neutral concepts. With the purpose of exploring the nature of such neutrality, we should take particularly into account the arguments behind two main opposite-based proposals: Atanassov's intuitionistic fuzzy sets [4] (proved to be equivalent to interval-valued fuzzy sets [20],[67],[74],[79] but also [13]) and Dubois-Prade bipolarities [22],[24] (see next section for a comparison between our paired approach and other related models).

Starting from a concept of interest $P$, a first observation is that a negation $N$ is given in our model, from which it is then possible to define different antonyms or subantonyms. In this sense, let us remark that $P$ and its negation not- $P$ contain the same information in our model (we should anyway remind that intuition works with positively defined concepts, see e.g. [21], but also [48]). In this sense, a predicate and its negation indeed constitute two paired predicates implying a particular empty space between opposites: no neutral concept can be defined in between a predicate and its negation, although estimation uncertainty can play a role, as already pointed out.

Anyway, as suggested in the preliminary example, neutrality may arise in different manners, whenever an object fails to be fully described from the two reference predicates. Neutrality will in fact represent a different concept from opposites, another relevant available option for evaluating objects. Particularly, different paired structures will exhibit different types of neutrality, in such a way that its nature will sometimes be definitive in order to identify the particular paired structure we are dealing with.

Although the objective of this paper is, as already pointed out, simply positional, let us grasp the formal model to be developed: starting from a basic predicate (and its negation) we can define an opposite that might imply, to some extent, the existence of indeterminacy (antonym) and/or ambivalence (sub-antonym), and also conflict. Hence, the point of departure for a general definition of a paired structure is given by a qualitative scale

$$
L=\{\text { concept }, \text { opposite; indeterminacy, ambivalence, conflict }\},
$$

composed by a pair of opposite concepts and three types of neutrality as primary valuations. This crisp setting corresponds to a basic model in which objects are evaluated by assigning exactly one concept to each object. In order to allow these valuations to be more general, it is then possible to introduce secondary valuations (referring to a secondary, ordered scale $\underline{S}$, for instance $S=[0,1]$ ) expressing the degree of fulfilment of each of the predicates acting as primary valuations, consistently with [2] and [48]. 
Thus, in a first formal approach, our paired structures can be represented through a multidimensional fuzzy set $A_{L}$ given by

$$
A_{L}=\left\{\left\langle x ;\left(\mu_{s}(x)\right)_{s \in L}\right\rangle \mid x \in X\right\},
$$

where $X$ is the universe of discourse and each object $x \in X$ is assigned up to a degree $\mu_{s}(x) \in S$ to each one of the above five predicates $s \in L$. This structure $\left(X, L, S, A_{L}\right)$ should be then provided with an appropriate logic (or logics, see [44],[45]), consistently enabling the composition of arguments.

Remark 1. Notice that we have adopted the usual valuation scale of fuzzy sets, the unit interval $[0,1]$, as the secondary scale $S$ expressing degrees of verification of the primary predicates in $L$. However, as pointed out in [33], any other complete lattice would be as valid meanwhile it allows representing the adequate gradation needed in each context. In this sense, the simplest option, corresponding to the previously mentioned crisp, binary setting, would be to take $S=\{0,1\}$. On the other extreme, if it is needed to represent imprecision in the estimated degrees, then it is possible to allow intervals (or even general type-2 fuzzy sets) as secondary valuations, leading to $S$ as the set of interval-valued fuzzy sets (or any other appropriate type-2 lattice).

Remark 2. Although we do not think that just one kind of formalism is enough to represent all the relationships between the valuation predicates in the primary scale $L$, we indeed think that it is crucial for such a structure to be somehow described and represented, i.e. specifying the valuation predicates and the relationships holding between them. In fact, one of our main objectives when writing this positional paper is to stress this structural issue. We should clarify what makes a predicate to be associated with its character besides its name. As we will be discussing in the next section, many of the existing extensions of fuzzy sets fail to describe any underlying structure, and as a consequence, it is usually difficult to appreciate what are the actual differences and similarities between those extensions. For instance, and following [48], the relationships between our primary predicates in $L$ can be explained in terms of a digraph, where neutrality with respect to our two opposites must be structurally acknowledged as neutral. But in some contexts these relationships might be better expressed through constraints relating the different degrees $\mu_{s}(x), s \in L$, so they reflect their intended meaning. In this sense, notice that, following [1] and [2], we are not in principle assuming that $\sum_{s \in L} \mu_{s}(x)=1$, which for each $x \in X$ would define a convex polyhedron in $[0,1]^{L}$. Specific constraints like this one might be appropriate depending on the chosen logical connectives (see [55],[65],[80]). Of course, other generalizations of fuzzy sets may also be considered for the representation of predicates (see, e.g., [11]).

\section{A comparison with some other opposite-based models}


In 1965 Zadeh introduced the notion of fuzzy set [85]. Since it appeared, it was clear that there was a problem for building the membership degree of each element in the considered set. For this reason, in 1971 he proposed the first idea of an extension, called type-2 fuzzy set (see [42],[86]). In this extension, the membership degree of each element is given by a fuzzy set of the valuation scale $[0,1]$. Furthermore, the problem of constructing the membership degrees has led to the introduction of different extensions, of which we highlight interval-valued fuzzy sets (also known as grey sets, and equivalent to vague sets), fuzzy multisets and $n$-dimensional fuzzy sets, neutrosophic sets, hesitant fuzzy sets, meta-fuzzy sets, etc. (see, e.g., [12]). There exist also other types of extensions (see, e.g., [13]), as probabilistic sets, fuzzy rough sets or fuzzy soft sets, which have a different origin from that of the previous ones, since they were not introduced to solve the problem of defining the membership degree of the elements. This large sequence of names and models has indeed produced certain confusion. In fact, some of those models were later found to be equivalent to existing ones, and in some cases the intuitive justification behind such models does not fully fit the mathematical model itself (some denominations happen to be equivalent only when certain structural issues are not taken into account, as pointed out in [48]). Some light should be brought to this problem if we address how the most basic knowledge representation models are generated from two opposite concepts. Hence, let us then comment the relationship between our paired approach and some of the main oppositebased models: interval valued fuzzy sets, shadowed sets, Atanassov's intuitionistic fuzzy sets, bipolar fuzzy sets, and neutrosophic fuzzy sets

Among all those opposite-based fuzzy models, Atanassov's intuitionistic fuzzy sets (A-IFS) deserve a particular analysis (see [21] for a complete analysis). According to Atanassov [4], an A-IFS assigns to each object both a degree of verification to certain property and a degree of verification to the negation of such a property, in such a way that these two degrees of verification sum at most 1 . In this way, a principle which is demanded in classical logic can be recovered: the Lukasiewicz conjunction of the degree of verification of a property and the degree of verification of the negation of that property is always 0 , and thus the non-contradiction law holds.

Moreover, Atanassov introduced the hesitancy or indeterminacy index as 1 minus the sum of the degrees of verification of the property and its negation. This index intends to represent the difficulty in the problem of building the exact membership and non-membership functions. Hence, a fuzzy partition in the sense of Ruspini [66] is being defined in terms of the given property, its negation and the remaining hesitancy.

First of all, it has been criticized that the entitlement "intuitionistic" looks inappropriate despite many scientists have accepted such a denomination (see the alternative intuitionistic fuzzy model proposed in [73]). Moreover, measuring the degree of verification of the negation of a property seems difficult to achieve in practice (for example, within a Probability framework we rarely ask for the probability of the negation of an event: we usually evaluate the probability of the event and then we reckon the probability of its negation... Otherwise most probably the values we provide for the event and its negation will not sum up to 1).

In addition, what the remaining category of "indeterminacy" means is not clear along Atanassov's texts. Anyway, as already pointed out, from a purely mathematical view A-IFS are equivalent to interval-valued fuzzy sets (IVFS), in such a way that the length of each interval is the same as the hesitancy index defined by Atanassov. 
Nevertheless, it has been pointed out in [48] that the structural issue is missing in both models. By explicitly stating the different predicates that intervene as well as the relationship between them, both models can be distinguished in the framework of paired structures (and particularly in that of meta-fuzzy sets, see [48]). Without addressing the structural issue, both Atanassov's intuitionistic fuzzy sets and intervalvalued fuzzy sets are simply defining a Ruspini's partitions [66] of three classes, no matter the different names we assign to each of the three categories in each model.

Beside Atanassov's argumentation, in our opinion the key element in his model is that he puts together several fuzzy sets, stressing in this way that we cannot evaluate each fuzzy set in an isolated way. A similar claim can be found in specific frameworks, see e.g. [46], where in order to escape from Arrow's paradox [3] different types of intensity preferences (strict preferences, weak preferences, indifferences, and potentially incomparability) were simultaneously amalgamated into structured Ruspini's partitions [66]. Ruspini's partition was generalized in [2] by suppressing any restriction on the degrees of membership, producing what Miyamoto [43] calls multiset (see also [83]). But notice that classes in [2] were expected to evolve in terms of nonoverlapping, covering and relevance (see [1]), consistently with our paired approach. In this sense it is interesting to point out that $n$-dimensional fuzzy sets [69] are multisets implying the existence of a linear structure for the $n$ classes under consideration.

On the other hand, the concept of bipolarity in a fuzzy framework was initially proposed by Zhang and Zhang (see [87], [88]), assigning two independent valuations to each object. As already pointed out above, the term bipolarity comes from Psychology where it has a specific meanings that might not properly fit neither into this proposal nor into the three types of bipolarity considered by Dubois and Prade (see again [22],[23],[24]). Zhang and Zhang assumed that one of the two opposites is assigned a value on the unit interval $[0,1]$, while the other one ranges in the opposite interval $[-1,0]$. Hence, since both intervals are isomorphic, and no matter the terms used to intuitively justify these two values, the fact is that Zhang-Zhang's bipolar model appears as a specific multiset $(n=2)$, with no restriction on their two degrees of membership. Zhang-Zhang's bipolar model is therefore equivalent to the neutrosophic sets proposed by Smarandache [70]. Notice also that none of these two equivalent models include any formal structure, as claimed in [48]. But the selected denominations within each model might suggest different underlying structures: while the model proposed by Zhang and Zhang suggests conflict between categories (a specific type of neutrality different from Atanassov's indeterminacy), Smarandache suggests a general neutrality that should perhaps jointly cover some of the specific types of neutrality considered in our paired approach.

Shadowed sets [57] are a particular case of interval-valued fuzzy sets, being isomorphic with a three valued logic: each object is assigned a " 0 " if certain property does not hold, " 1 " if it does hold, and the unit interval $[0,1]$ otherwise. Anyway, it might be unclear the meaning of the selected term for this third stage: "complete ignorance" might refer to an extremely poor estimation or to extremely poor knowledge about the model itself, for example.

All in all, the above discussion suggests that there is an urgent need to put some unifying light on the similarities and differences of all the mentioned extensions of fuzzy sets in terms of their representational structures and capabilities. This is precisely the idea we wish to communicate through this positional paper on paired structures: the 
need for a unifying framework in which the different models arise as a consequence of the specified structure (on the primary predicates) and the different secondary scales. Such a unifying view should then allow a deeper analysis of the relationships between several knowledge representation formalisms, providing a basis from which more expressive models may be later developed.

Summing up, the structural issue is required for a proper specification of meaning in a logical-mathematical modelling. If structural issues are not properly addressed in our mathematical models, we might be easily confused between different approaches once they appear as isomorphic, simply because the difference in structure is not taken into account. This was the main issue raised in [48], in this paper focussed into opposite-based models. Our mathematical models should capture all the essential aspects of reality, including the relations between modelled predicates. Listing elements should be accompanied with the relations between the elements of that list. It is the structural difference what justifies a denomination, not the other way around. Structural performance of a set of concepts does not come with a list of unrelated objects or names. If these elements suggest a structure, such structure should be formally stated.

\section{$5 \quad$ Building Paired Structures}

In our opinion, the most basic structure to represent knowledge should at least contain a predicate and its opposite, particularly in terms of negation, antonym or sub-antonym. In this way we can distinguish an arbitrary couple of concepts from two paired predicates, and the associated neutral concepts naturally appear from the characteristics of such an opposition. On this basis, we can propose a basic approach to a standard procedure for building paired structures:

1) We always start from a concept and its negation (a must in our model, since antonym or sub-antonym are relative to the given negation).

2) Then, any two opposite predicates are paired concepts and, if different from such a negation, their semantic relation will generate additional and specific neutral concepts. In case our original predicate and its opposite do not overlap, indeterminacy arises (our paired predicates do not fully explain reality, and they can be reshaped into wider concepts or they can suggest a search for additional information, producing perhaps a new intermediate non-neutral concept). In case our seminal predicate and its opposite overlap, ambivalence arises (the existence of a new concept associated to such overlapping is suggested together with a reshaping of opposites into more precise concepts). Of course indeterminacy and ambivalence can appear simultaneously, whenever the opposition is neither antonym nor sub-antonym (overlapping in some objects might suggest ambivalence, and lack of fulfilment in some other objects might suggest indeterminacy).

3) Whenever we detect conflict, it entails that the considered paired concepts are viewed in our mind as complex concepts that should be decomposed into and aggregated from simpler concepts. In addition, each one of those simpler concepts is subject to the previous arguments (the concept, its negation, its antonym or its subantonym, etc.) 
Hence, three main families of paired fuzzy sets appear in this paper:

- Those "basic" paired concepts based upon the negation of both predicates, with no additional neutral concept being allowed.

- Those "simple" paired concepts based upon opposites (antonym or subantonym associated to indeterminacy or ambivalence neutralities, and perhaps both simultaneously).

- And those "complex" paired concepts based upon multidimensionality, where in addition to indeterminacy and ambivalence we can find different levels of conflict.

These three families of paired fuzzy sets are close to the three types of bipolarities proposed by Dubois and Prade in [22],[23],[24]. But rather than focusing on the formalization of scales that address some representational issues when dealing with poles, our focus is on the configuration of such poles through opposition relationships, and how these determine the scales to be used regarding the representational power of the models.

\section{Final Comments}

In this paper we have presented a systematic approach to different types of paired sets, to be considered as an alternative to the notion of bipolarity proposed by Zhang and Zhang ([87], [88]), Dubois and Prade ([22], [23], [24]) and other proposals somehow based upon two basic opposite concepts (but taking into account the structural arguments of [48]). All of these models are deeply related to Atanassov's intuitionistic fuzzy sets (see [4], [5]).

The main aim of this paper is to bring some light into this discussion, bolding the constructive argument towards a unifying view and stressing the role of different neutralities in between two opposite concepts. In particular, we postulate that paired structures represent a basic model for most learning processes, which quite often start from two opposite predicates (see [28], [47]). Relation between these two predicates can create specific neutral concepts: indeterminacy (which can be justified in terms of too narrow concepts), ambivalence (which can be justified in terms of too wide opposites), and conflict (which can be justified in terms of multidimensional opposites). But it is the specific semantic tension between two basic opposites the key aspect to be analysed. Of course the three different neutralities we have considered in this paper can appear in the same problem, together with other uncertainties (particularly, imprecision). How we can simultaneously manage all these parameters becomes a suggesting and necessary objective for future research.

In order to stress its unifying view on a number of models that are based upon the existence of two opposites, a full mathematical development of our paired structures have been left outside this positional paper. A first application of our paired approach has been presented in [32]. Another interesting field to consider next should be Sentiment Analysis, where indeed two opposite meanings (positive, negative) are associated to terms within a text that can be classified according our approach (see, e.g., 
[56]). Also, as already pointed out, main issues to be addressed in future research on paired structures concern the study of different formalisms to represent the relationships between the elements of the structure (e.g. sets of logical constraints on the degrees of the qualitative predicates, digraphs, partial orders, etc.) and their effect in relation with the representational power of the associated structures.

A possible drawback of the general case of paired fuzzy structures is that they can be considered too complex for some applications, since it might imply the direct estimation of quite a number of degrees of verification for each object (in particular when our concepts have a multicriteria nature and all neutralities are present). Moreover, our structure has to be completed addressing imprecision. In order to manage this complexity, another main issue for future research on paired structures should be to explore other extensions of fuzzy sets (see, e.g., [15]). An alternative approach is to obtain some degrees of membership or membership functions from a smaller subset of categories, in such a way that the whole system can be fully described from a few parameters to be estimated. To some extent, this is similar to what is sometimes done in preference modelling, where three neutralities are naturally learnt: the preferences between two alternatives may (they are perhaps weak preferences, leading to the ambivalent indifference), or they are perhaps very extreme strict preferences (leading to indeterminacy); or they can even reflect some multicriteria-based conflict (sometimes solved in terms of incomparability, see e.g. [30], [31], [50] and particularly [46]). Similar models are found concerning different extensions of Belnap's logic [8], like that proposed in [77] (see also [55], [58] and [65], [80]). The study of appropriate logic connectives will play a crucial role in constructing and constraining the semantics of the different predicates generated from the two reference opposites (see also [26]). In this sense, aggregation operators play a crucial role in all these models, and they should play a similar role in relation to complex paired structures in which multidimensional concepts are decomposed into (and obtained from) simpler concepts. Moreover, as suggested in introduction, another interesting objective for future research is to extend this model into those multiple concepts that cannot be understood with a unique opposite concepts, but they need several concepts to capture its limits.

All in all, the main objective of this paper is to stress the need for a unifying framework that should allow a better understanding of different models in terms of different specifications of a basic, quite general structure. Such a unifying view should then allow a clarifying analysis of the relationships between several knowledge representation formalisms. A basis for the development of more expressive models should be also provided.

In particular, paired structures stand as a first attempt to describe reality through the logical representation of context and the paired aggregation of semantically oppositerelated concepts. As a point of departure for knowledge representation, paired structures allow building the meaning of concepts by logical analysis, learning their semantic structure. The semantical relationship between paired concepts can suggest new concepts or their own reshaping. Paired fuzzy sets and paired structures should be considered a basic model to move on towards more accurate representation models.

\section{Acknowledgements}


This research has been partially supported by the Government of Spain (grants TIN2012-32482 and TIN2013-40765-P), the Government of Madrid (grant S2013/ICCE-2845) and the UCM (Research Group 910149). We want to express our gratitude to Prof. Didier Dubois for sharing some time ago his critical view towards a preliminary version of our approach, which indeed has helped us to clarify our position. We also thank the anonymous reviewers for their positive comments that helped to improve the paper in key aspects.

\section{References}

[1] A. Amo, D. Gómez, J. Montero, G. Biging: Relevance and redundancy in fuzzy classification systems. Mathware and Soft Computing 8 (2001), 203-216.

[2] A. Amo, J. Montero, G. Biging, V. Cutello. Fuzzy classification systems. European Journal of Operational Research 156 (2004), 495-507.

[3] K.J. Arrow: Social Choice and Individual Values (Yale University Press, 1951 first edition).

[4] K.T. Atanassov: Intuitionistic fuzzy sets, Fuzzy Sets and Systems 20 (1986), 87-96.

[5] K.T. Atanassov: Answer to D. Dubois, S. Gottwald, P. Hajek, J. Kacprzyk and H. Prade's paper "Terminological difficulties in fuzzy set theory - the case of "Intuitionistic fuzzy sets". Fuzzy Sets and Systems 156 (2005), 496-499.

[6] A. Bechara, D. Tranel, H. Damasio: Characterization of the decision-making deficit of patients with ventromedial prefrontal cortex lesions. Brain 123 (2000), 2189-2202.

[7] A. Bechara, H. Damasio, A.R. Damasio: Role of the amygdala in decision-making. Annals of the New York Academy of Sciences 985 (2003), 356-369.

[8] N.D. Belnap: A useful four-valued logic. In: G. Epstein and J. Dumme (eds.), Modern uses of multiple valued logics (Reidel, Dordrecht, 1977).

[9] J.Y. Beziau, D, Jacquette (eds.): Around and beyond the square of opposition (Birkhäuser, 2012).

[10] E.D. Booman, M.F.S. Rushworth, Conceptual representation and the making of new decisions. Neuron 63 (2009), 721-723.

[11] M. Bunge: Emergence and convergence: Qualitative novelty and the unity of knowledge (University of Toronto Press, 2003).

[12] H. Bustince, E. Barrenechea, J. Fernández, M. Pagola, J. Montero: The Origin of Fuzzy Extensions. In B. De Baets, R. Mesiar (eds.): Springer Handbook of Computational Intelligence (Springer-Verlag, Berlin, 2015); pp. 89-112.

[13] H. Bustince, E. Barrenechea, M. Pagola, J. Fernandez. Z. Xu, B. Bedregal, J. Montero, H. Hagras, F. Herrera, B. De Baets: A historical account of types of fuzzy sets and their relationships. IEEE Transactions on Fuzzy Systems (in press).

[14] H. Bustince, J. Fernandez, R. Mesiar, J. Montero, R. Orduna: Overlap functions. Nonlinear Analysis 72 (2010), 1488-1499.

[15] H. Bustince, F. Herrera, J. Montero (eds.): Fuzzy Sets and their extensions, representation, aggregation and models. Studies in Fuzziness and Soft Computing 220 (2008).

[16] H. Bustince, M. Pagola, E. Barrenechea, J. Fernandez, P. Melo-Pinto, P. Couto, H.R. Tizhoosh, J. Montero: Ignorance functions. An application to the calculation of the threshold in prostate ultrasound images. Fuzzy Sets and Systems 161 (2010), 20-36.

[17] J.T Cacioppo, G.G. Berntson: The affect system, architecture and operating characteristics - Current directions. Psycological Science 8 (1999), 133-137.

[18] J.T. Cacioppo, W.L. Gardner, C.G. Berntson: Beyond bipolar conceptualizations and measures - The case of attitudes and evaluative space. Personality and Social Psychology Review 1 (1997), 3-25. 
[19] B. De Baets, R. Mesiar: T-partitions. Fuzzy Sets and Systems 97 (1998), 211-223.

[20] G. Deschrijver, E.E. Kerre: On the position of intuitionistic fuzzy set theory in the framework of theories modelling imprecision. Information Sciences 177 (2007), 1860-1866.

[21] D. Dubois, S. Gottwald, P. Hajek, J. Kacprzyk, H. Prade: Terminological difficulties in fuzzy set theory - the case of intuitionistic fuzzy sets. Fuzzy Sets and Systems 156 (2005), 485-491.

[22] D. Dubois, H. Prade: An introduction to bipolar representations of information and preference, International Journal of Intelligent Systems 23 (2008), 866-877.

[23] D. Dubois, H. Prade: An overview of the asymmetric bipolar representation of positive and negative information in possibility theory. Fuzzy Sets and Systems 160 (2009), 1355-1366.

[24] D. Dubois, H. Prade: Gradualness, uncertainty and bipolarity - Making sense of fuzzy sets, Fuzzy Sets and Systems 192 (2012), 3-24.

[25] D. Dubois, H. Prade: From Blanché's hexagonal organization of concepts to formal concept analysis and possibility theory. Logica Universalis 6 (2012), 149-169.

[26] J. Dujmovic: Aggregation operators and observable properties of human reasoning. Advances in Intelligent Systems and Computing 228 (2013), 5-16.

[27] J. Edwards, T. Ostrom. Cognitive structure of neutral attitudes. Journal of Experimental Social Psychology 7 (1971), 36-47.

[28] M. Espinilla, J. Montero, J.T. Rodríguez: Computational intelligence in decision making. International Journal of Computational Intelligence Systems 6, Supp. 2 (2013), 1-5.

[29] J. Fodor, B. de Baets, P. Perny: Preferences and decisions under incomplete knowledge (Physica, 2013).

[30] J. Fodor, M. Roubens: Fuzzy Preference Modelling and Multicriteria Decision Support (Kluwer Academic Publishers, Dordrecht, 1994).

[31] C. Franco, J. Montero, J.T. Rodríguez: A fuzzy and bipolar approach to preference modeling with application to need and desire. Fuzzy Sets and Systems 214 (2013), 20-34.

[32] C. Franco, J.T. Rodríguez, J. Montero: Building the meaning of preference from logical paired structures. Knowledge-Based Systems 83 (2015), 32-41.

[33] J.A. Goguen: Journal of Mathematical Analysis and Applications 18 (1967), 145-174.

[34] J. González-Pachón, D. Gómez, J. Montero, J. Yáñez: Soft dimension theory. Fuzzy Sets and Systems 137 (2003), 137-149.

[35] J. González-Pachón, D. Gómez, J. Montero, J. Yáñez: Searching for the dimension of valued preference relations. International Journal of Approximate Reasoning 33 (2003), 133-157.

[36] M. Grabisch, S. Greco, M. Pirlot. Bipolar and bivariate models in multi-criteria decision analysis: descriptive and constructive approaches. International Journal of Intelligent Systems 23 (2008), 930-969.

[37] M. Grabisch, Ch. Labreuche: Bi-capacities for decision making on bipolar scales. Proceedings EUROFUSE Workshop in Information Systems (2002), 185-190.

[38] K. Kaplan: On the ambivalence-indifference problem in attitude theory and measurement A suggested modification of the semantic differential technique, Psychological Bulletin 77 (1972), 361-372.

[39] D. Kumaran, J.J. Summerfield, D. Hassabais, E.A. Maguire: Tracking the emergence of conceptual knowledge during human decision making. Neuron 63 (2009), 889-901.

[40] R. Likert: A technique for the measurement of attitudes. Archives of Psychology 140 (1932), $1-55$.

[41] M.L. Mack, A.R. Preston, B.C. Love: Decoding the Brain's Algorithm for Categorization from Its Neural Implementation. Current Biology 23 (2013), 2023-2027.

[42] J.M. Mendel: Advances in type-2 fuzzy sets and systems. Information Sciences 177 (2007), 84-110.

[43] S. Miyamoto: Multisets and fuzzy multisets. In Z.Q. Liu, S. Miyamoto (eds.): Soft Computing and Human-Centered Machines (Springer, Berlin, 2000); pp. 9-33.

[44] J. Montero: Comprehensive fuzziness. Fuzzy Sets and Systems 20, 79-86 (1986). 
[45] J. Montero: Extensive fuzziness. Fuzzy Sets and Systems 21, 201-209 (1987).

[46] J. Montero: Arrow's theorem under fuzzy rationality. Behavioral Science 32 (1987), 267273.

[47] J. Montero: Fuzzy logic and science. Studies in Fuzziness and Soft Computing 243 (2009), 67-78.

[48] J. Montero, D. Gómez, H. Bustince: On the relevance of some families of fuzzy sets. Fuzzy Sets and Systems 158 (2007), 2429-2442.

[49] J. Montero, V. López, D. Gómez: The role of fuzziness in decision making. Studies in Fuzziness and Soft Computing 215 (2007), 337-349.

[50] J. Montero, J. Tejada, V. Cutello: A general model for deriving preference structures from data. European Journal of Operational Research 98 (1997), 98-110.

[51] P. Murinova, V. Novak: Analysis of generalized square of opposition with intermediate quantifiers. Fuzzy Sets and Systems 242 (2014), 89-113.

[52] N. Naqvi, B. Shiv, A. Bechara: The role of emotion in decision making, a cognitive neuroscience perspective. Current Directions in Psychological Science 15 (2006), 260-264.

[53] V. Novak: A comprehensive theory of trichotomous evaluative linguistic expressions. Fuzzy Sets and Systems 159 (2008), 2939-2969.

[54] C.E. Osgood, G.J. Suci, P.H. Tannenbaum: The measurement of meaning (University of Illinois Press, Urbana, 1957).

[55] M. Ozturk, A. Tsoukias: Modelling uncertain positive and negative reasons in decision aiding. Decision Support Systems, 43 (2007), 1512-1526.

[56] N. Pang, L. Lee: Opinion mining and sentiment analysis. Foundations and Trends in Information Retrieval 2 (2008), 1-135.

[57] W. Pedrycz: Shadowed sets, representing and processing fuzzy sets. IEEE Transactions on Systems, Man and Cybernetics B 30 (2000), 249-262.

[58] P. Perny, A. Tsoukiàs: On the continuous extensions of four valued logic for preference modelling. In B. Bouchon-Meunier (ed.): Proceedings of the IPMU conference (Editions E.D.K., Paris, 1998), 302-309.

[59] J. Peters, C. Büchel: Episodic future thinking reduces reward delay discounting through an enhancement of prefrontal-mediotemporal interactions. Neuron 66 (2010), 138-148.

[60] J. Peters, C. Büchel: The neural mechanisms of inter-temporal decision-making: understanding variability. Trends in Cognitive Sciences, 15 (2011), 227-235.

[61] J.W. Reich, A.J. Zautra, P.T. Potter: Cognitive structure and the independence of positive and negative affect. Journal of Social and Clinical Psychology 20 (2001), 99-115.

[62] J.T. Rodríguez, C. Franco, J. Montero: On the semantics of bipolarity and fuzziness. Advances on Intelligent and Soft Computing 107 (2011), 193-205.

[63] J.T. Rodríguez, C. Franco, J. Montero, J. Lu: Paired Structures in Logical and Semiotic Models of Natural Language. In A. Laurent, O. Strauss, B. Bouchon-Meunier, R.R. Yager (eds.): Information Processing and Management of Uncertainty in Knowledge-Based Systems, Part II (2014); pp. 566-575.

[64] J.T. Rodríguez, C. Franco, B. Vitoriano, J. Montero: An axiomatic approach to the notion of semantic antagonism. In K. Hirota, M. Mukaidono, S. Kuswadi (eds.): Proceedings IFSAAFSS Conference, Surabaya \& Bali, Indonesia, June 21-25 (2011) FT104-1/6.

[65] J.T. Rodríguez, E. Turunen, D. Ruan, J. Montero: Another paraconsistent algebraic semantics for Lukasiewicz-Pavelka logic. Fuzzy Sets and Systems 242 (2014), 132-147.

[66] E.H. Ruspini: A new approach to clustering. Information and Control 15 (1969), 22-32.

[67] R. Sambuc: Fonctions $\varphi$-flues. Application á l'aide au diagnostic en pathologie thyroidienne, Ph.D. Thesis (Université de Marseille, 1975).

[68] G. Shafer: A mathematical theory of evidence (Princeton University Press, NJ, 1976).

[69] Y. Shang, X. Yan, E.S. Lee: The n-dimensional fuzzy sets and Zadeh fuzzy sets based on the finite values sets. Computer and Mathematics with Applications 60 (2010), 442-463.

[70] F. Smarandache: A unifying field in logics, neutrosophic logic, neutrosophy, neutrosphic 
set, neutrosophic probability and statistics (American Research Press, 2005).

[71] P. Slovic, E. Peters, M.L. Finucane, D.G. MacGregor: Affect, risk, and decision making. Health Psychology 24 (2005), 35-40.

[72] A.R. de Soto, E. Trillas: On antonym and negate in fuzzy logic. International Journal of Intelligent Systems 14 (1999), 295-303.

[73] G. Takeuti, S. Titani: Intuitionistic fuzzy logic and intuitionistic fuzzy set theory. Journal of Symbolic Logic 49 (1984), 851-866.

[74] H.R. Tizhoosh: Interval-valued versus intuitionistic fuzzy sets, isomorphism versus semantics. Pattern Recognition 41 (2008), 1812-1813.

[75] E. Trillas: On the use of words and fuzzy sets. Information Sciences 176 (2006), 1463-1487.

[76] E. Trillas, C. Moraga, S. Guadarrama, S. Cubillo, E. Castineira: Computing with antonyms. Studies in Fuzziness and Soft Computing 217 (2007), 133-153.

[77] A. Tsoukiàs: A first-order, four valued, weakly paraconsistent logic and its relation to rough sets semantics. Foundations of Computing and Decision Sciences 12 (2002) 85-108.

[78] A.Tsoukiàs, Ph. Vincke: An axiomatic foundation of partial comparability theory. Theory and Decision 39 (1995), 79-114.

[79] I.B. Türksen: Interval valued fuzzy sets based on normal forms. Fuzzy Sets and Systems 34 (1986), 191-210.

[80] E. Turunen, M. Öztürk, A. Tsoukiás: Paraconsistent semantics for Pavelka style fuzzy sentential logic. Fuzzy Sets and Systems 161 (2010), 1926-1940.

[81] A. Tversky, D. Kahneman. Advances in prospect theory - cumulative representation of uncertainty. Journal of Risk and Uncertainty 5 (1992), 297-323.

[82] M.E. Walton, J.T. Devlin, M.F.S. Rushworth: Interactions between decision making and performance monitoring within prefrontal cortex. Nature Neuroscience 7 (2004), 12591265.

[83] R.R. Yager: On the theory of bags. International Journal of General Systems 13 (1986), 2337.

[84] J. Yáñez, J. Montero: A poset dimensión algorithm. Journal of Algorithms 30 (1999), 185208.

[85] L.A. Zadeh: Fuzzy sets. Information and Control 8 (1965), 338-353.

[86] L.A. Zadeh: The concept of a linguistic variable and its application to approximate reasoning. Information Sciences 8 (1975), 199-249.

[87] W.R. Zhang: NPN fuzzy sets and NPN qualitative algebra, a computational framework for bipolar cognitive modelling and multiagent decision analysis. IEEE Transactions on Systems, Man and Cybernetics B, 26 (1996), 561-574.

[88] W.R. Zhang, L. Zhang: YinYang bipolar logic and bipolar fuzzy logic. Information Sciences 165 (2004), 265-287. 